\title{
Simultaneous Measurements of the Liquid Phase Velocity and Gas Bubble Trajectories in a Two-Phase Flow at Gas- Evolving Electrodes
}

\author{
D. Baczyzmalski ${ }^{1}, T$. Weier ${ }^{2}$, C.J. Kähler ${ }^{1}$, C. Cierpka ${ }^{1}$ \\ ${ }^{1}$ Institute of Fluid Mechanics and Aerodynamics, Universität der Bundeswehr München, \\ 85577 Neubiberg, Germany, \\ Dominik.Baczyzmalski@unibw.de \\ ${ }^{2}$ Helmholtz-Zentrum Dresden - Rossendorf, Institute of Fluid Dynamics, 01328 Dresden, Germany
}

\begin{abstract}
:
In water electrolysis the efficiency is related to the free area of the electrodes, which is limited by hydrogen gas bubbles evolving at the cathode. Therefore, a fast transport of the hydrogen bubbles away from the electrodes into the bulk is beneficial. The detachment and transport of bubbles from the electrode is affected by the convection of the electrolyte surrounding the bubble. In order to characterize the flow close to the electrode's surface, simultaneous measurements of the fluid velocities of the electrolyte and the size and trajectories of hydrogen bubbles were carried out in an undivided water electrolyzer at the Helmholtz-Zentrum Dresden - Rossendorf. The liquid phase velocity was measured by particle image velocimetry (PIV) as well as particle tracking velocimetry (PTV) using fluorescent tracer particles and laser light illumination. Gas bubble trajectories were determined using particle tracking on bubble shadow images obtained by a second camera in conjunction with a background illumination. The images were separated by the different wavelengths of the illumination/fluorescence. However, the uncertainty of the fluid and gas bubble velocity is strongly affected by the high void fraction in the vicinity of the electrode. As a result, liquid phase velocities determined with PIV are substantially biased in the near-wall region. The application of advanced PTV methods allows for more precise measurements closer to the electrode. Thus, an improved characterization of the evolving wall jet can be achieved by a combination of both PIV and PTV methods. Furthermore, the effect of large rising bubble with path oscillations on fluid velocities has been investigated. The wake generated by these bubbles can have a strong impact on the nearwall fluid velocities and their fluctuations and thus influence the mass transfer near the electrodes and the performance of the water electrolyzer.
\end{abstract}

Key words: two-phase flow, gas bubbles, particle tracking velocimetry, particle image velocimetry, water electrolysis

\section{Introduction}

Renewable energy technologies play an increasingly important role in order to limit the worldwide rising $\mathrm{CO}_{2}$ emissions. Moreover, the promotion of volatile energy sources such as solar or wind energy will be accompanied by the need for efficient energy storage systems. In particular, high expectations are placed on hydrogen as a promising energy storage system with a high energy density in comparison to mechanical energy storage technologies. Water electrolysis is an important technology for the production of hydrogen with the help of electric energy. However, the efficiency of this process is related to the amount of hydrogen and oxygen gas bubbles evolving at the electrode of the electrolyzer. As these gas bubbles have a very low electric conductivity, their presence lead to a reduction of the electrode's active area and an increase in the ohmic voltage drop across the electrode gap [4], [5]. It was found by several authors that a faster removal of the hydrogen gas bubbles from the electrode's surface into the bulk is useful to reduce the fractional bubble coverage and enhance the efficiency of the process. Moreover, it was realized that the detachment of bubbles from the electrode's surface is strongly affected by the convection of the surrounding electrolyte. Therefore, high interest has recently been shown in the application of forced convection, in particular by means of Lorentz forces generated by superimposed magnetic fields (see, e.g., [5]). However, only few experimental studies have been conducted to clarify the flow phenomena occurring in the 
two-phase flow of gas-evolving electrodes [1], [7]. The fluid velocities in the electrode nearregion are of particular interest to understand the mechanisms affecting bubble growth and detachment.

In order to investigate the underlying phenomena and thus help to enhance the performance of the electrolyzer, simultaneous measurements of the liquid phase velocity and bubble trajectories were performed in an undivided electrolysis cell with a vertical electrode setup. Special measuring and evaluation techniques had to be applied for reliable measurements in the vicinity of the electrode, where the void fraction is very high. Liquid phase velocities have been measured by using particle image velocimetry (PIV) and, in particular in the near-wall region, by particle tracking velocimetry (PTV). In addition, shadowgraphy was applied to simultaneously determine the trajectory of large rising bubbles and investigate their effect on the near-wall flow.

\section{Experiment}

A vertical arrangement of two stainless steel electrodes $\left(140 \times 25 \mathrm{~mm}^{2}[\mathrm{H} \times \mathrm{W}]\right)$ embedded in plastic holders was placed in a glass vessel, which was filled with a $0.25 \mathrm{M} \mathrm{NaOH}$ solution (see Fig.1). The distance between the electrodes was set to $30 \mathrm{~mm}$. A fixed current was applied to the electrodes to drive the water electrolysis process. The chemical reactions that take place at the electrodes' surface result in $\mathrm{O}_{2}$ and $\mathrm{H}_{2}$ gas bubbles evolving at the anode and cathode, respectively. These bubbles continue to grow until they are able to detach from the surface depending on their buoyancy, surface tension and convection of the surrounding electrolyte. Released bubbles rise from thereon close to the electrodes forming a confined layer of bubbles, i.e. the so-called bubble curtain (see right hand side of Fig. 1).

In order to measure the liquid phase velocity, fluorescent tracer particles doped with Rhodamin B (RhB, 1-20 $\mu \mathrm{m})$ were added to the solution. A solid state laser (532 nm, $1 \mathrm{~W})$ was used to align a vertical laser light sheet between both electrodes, which excited the fluorescent particles in that plane. Images of the light emitted by these tracers were recorded by a sCMOS camera (LaVision Imager sCMOS). A background illumination operating in the blue wavelength range (Lumencor SPECTRA X light engine) was used to take bubble shadow images with a second sCMOS camera simultaneously. Both cameras were equipped with different wavelength filters to separate the particle and bubble shadow images. A long pass filter (red filter in Fig. 1) was used to obtain only the particle images without distortions from the backlighting and the laser light reflected by the bubbles and walls. Similarly, a band pass filter (blue) allowed for the recording of only the bubble shadow image and blocking the laser reflections as well as the light emitted by the fluorescent tracer particles. This way, bubble shadow and tracer particles images were simultaneously recorded at $80 \mathrm{fps}$ with an optical magnification of 0.22 and a resolution of $34 \mathrm{pixel} / \mathrm{mm}$.

\section{Liquid Phase Velocity Measurements}

The velocity determination with PIV is based on the correlation of particle images of two consecutive frames, which are generally divided into smaller interrogation windows (see Fig. 2).
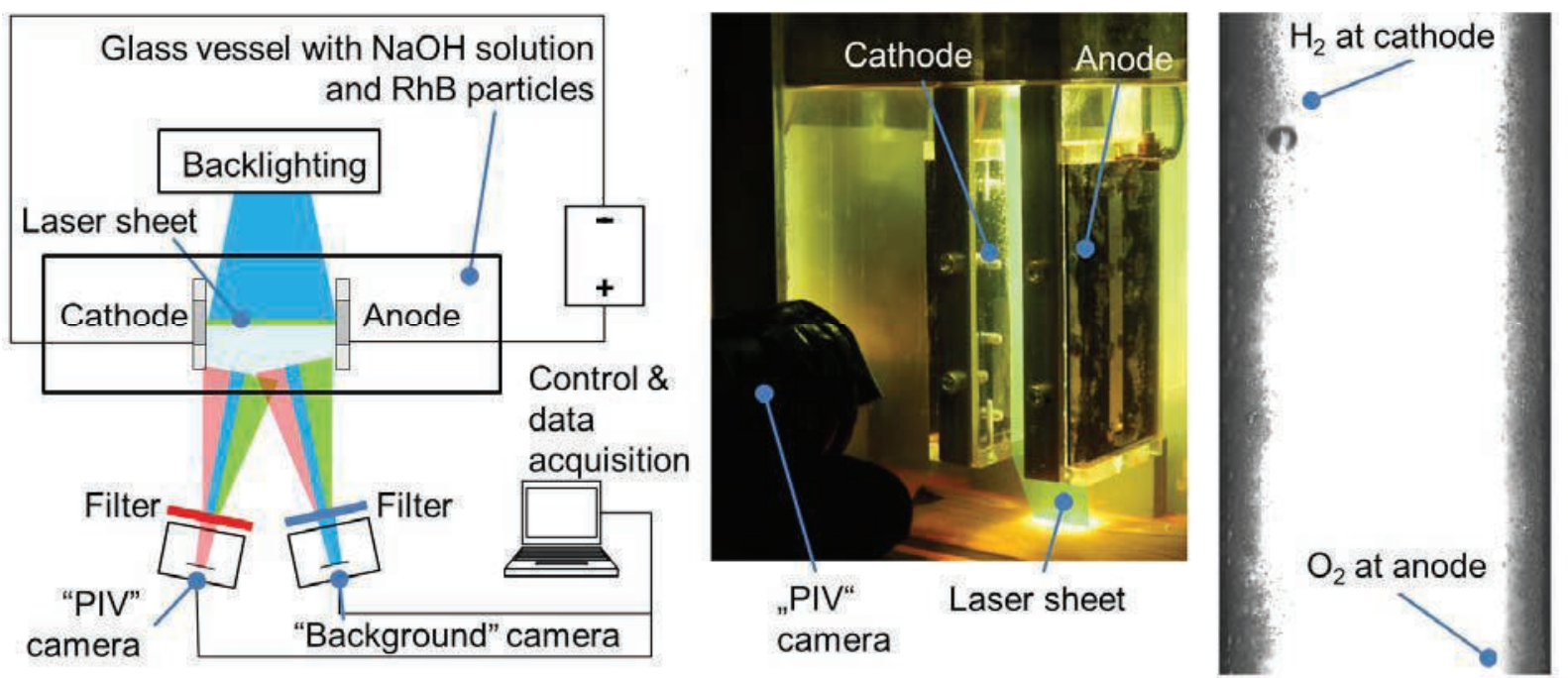

Fig. 1. Sketch of experimental setup (left). Photograph of setup (center). Bubble shadow image showing oxygen and hydrogen gas bubbles evolving at the anode and cathode, respectively (right). 


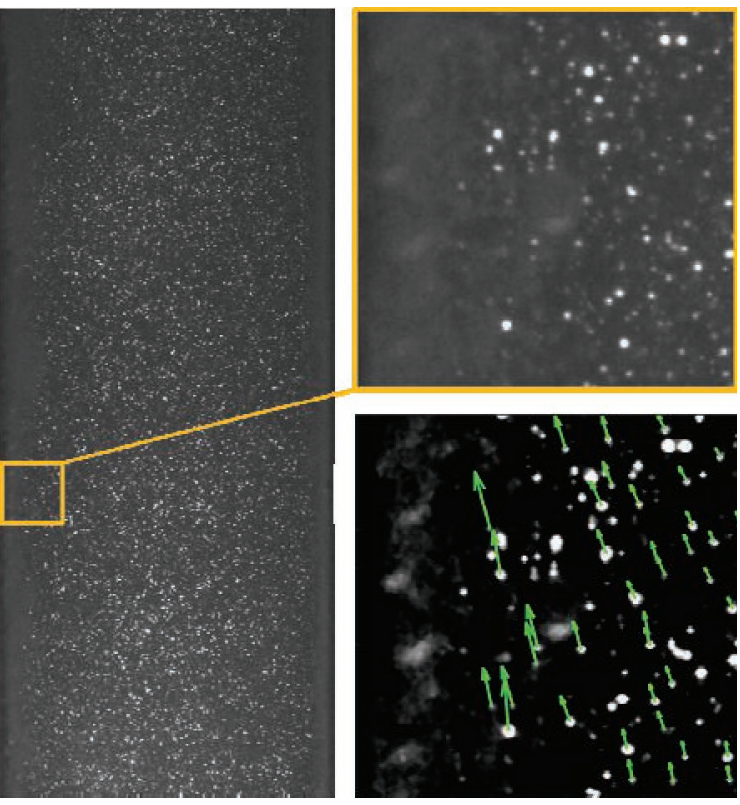

Fig. 2. Particle images used for PIV (left). Enlarged area close the cathode (top right) and corresponding image used for PTV with vectors determined by PTV, illustrating the displacement of particles (bottom right).

The average particle displacement in these windows can then be determined from the position of the corresponding correlation peak. Here, we used adaptive interrogation windows with a final size of $24 \times 24$ pixels with an overlap of $50 \%$.

As can be seen from Fig. 2, the void fraction in the near-wall region is so high that a large part of the light emitted by the tracer particles is blocked, which results in fluctuating and disappearing particle image intensities. It will be shown that this significantly biases the correlation result and thus the velocity determination in the near-wall region. However, an improved near-wall velocity determination could be obtained by using an advanced particle tracking scheme (see Fig. 2 bottom right). In contrast to PIV, the measuring accuracy of PTV does not depend on the particle image intensity itself but on the ability to detect and track these particles reliably. Different image filters were applied to remove the background noise and enhance the detectability of the particle images (see Fig. 2 bottom right). Nonetheless, spurious vectors will occur if the particle images become undetectable in one of the consecutive frames as a result of the high light absorption. In order to account for this and avoid wrong particle image pairing, an advanced tracking algorithm proposed by Ohmi and Lee (2000) [6] was applied, which compares the possible displacement of neighboring particles and excludes vectors which fall below a predetermined probability level [2]. In addition,

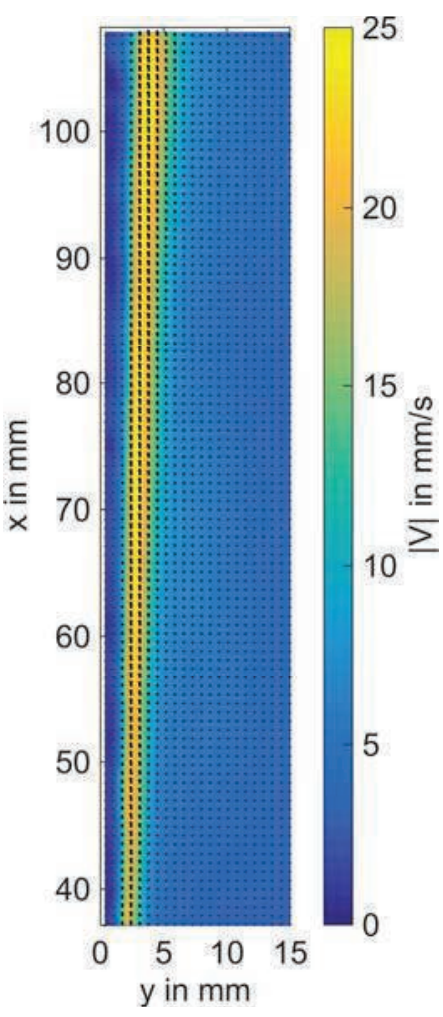

Fig. 3. Time-averaged PIV velocity field on the cathodic side for a current density of $j=1000 \mathrm{Am}^{-2}$. The origin of the coordinate system lies at the bottom of the cathode. The $x$ and $y$-axis are the vertical position along the cathode and the wall-normal distance from the cathode, respectively.

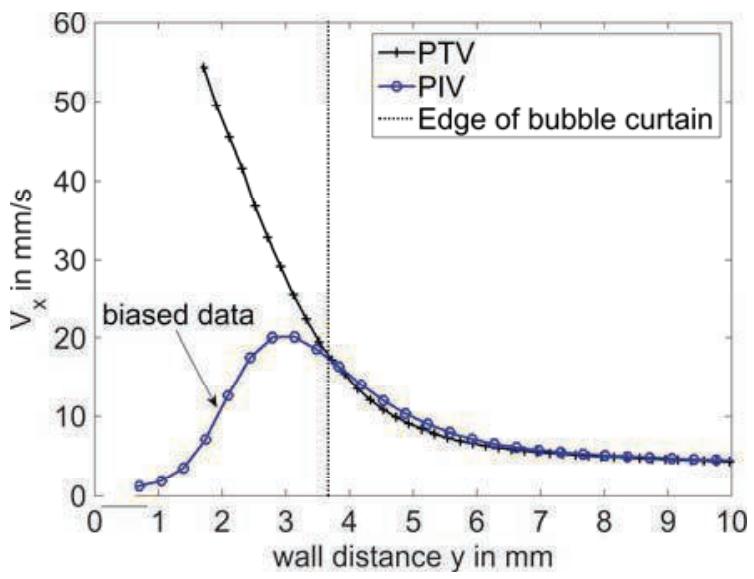

Fig. 4. Comparison of the PIV and PTV time and space-averaged wall parallel velocity components at the cathode for a current density of $j=1000 \mathrm{Am}^{-2}$. The edge of the bubble curtain was estimated from averaged bubble shadow images.

the universal outlier detection by Duncan et al. (2010) [3] was used to further reduce the amount of spurious vectors. Fig. 3 depicts PIV velocity field on the cathodic side averaged over a time period of $75 \mathrm{~s}$ (6000 images). Naturally, high velocities occur only near the cathode, where the flow is driven by the curtain of rising bubbles. However, the near-wall 
velocities are significantly biased as can be seen from Fig. 4, where the wall parallel velocities obtained by PIV and PTV were additionally averaged along the $x$-axis. The discrepancy between the PIV and PTV results starts at the edge of the light-absorbing bubble curtain and increases towards the electrode, as the void fraction and the light absorption becomes larger. Too few particles were found below $\mathrm{y}=1.5 \mathrm{~mm}$ to obtain reliable PTV results. However, the presented PTV approach allowed for more precise measurements closer to the electrode and thus an improved characterization of the near-wall velocity distribution, which is of high interest for the detachment of gas bubbles from the electrode surface.

\section{Effect of Large Bubbles}

The light absorption in the bubble curtain was too high to measure the velocity of bubbles moving within the curtain (see right hand side of Fig. 1). However, some large hydrogen bubbles $(\mathrm{d}=1-3 \mathrm{~mm})$ with path oscillations have been observed rising close to the edge of the bubble curtain (see, e.g., Fig. 5 right hand side). These bubbles evolve at the junction between the electrode and its holder, where they remain for a very long time and agglomerate to larger bubbles. This is a particular phenomenon of the current design and may not be present in other electrolyzes. However, the presence of these bubbles is of interest as they have an impact on the fluid velocities and mass transfer close to the electrode and thus influence the performance of the whole process.

A simple particle tracking algorithm and different image filters were used to determine the velocity and size of large rising bubbles. Fig. 5 shows the wake and trajectory of a single oscillating bubble with a diameter of $2 \mathrm{~mm}$ and an average wall parallel velocity of approximately $190 \mathrm{~mm} / \mathrm{s}$, which is substantially faster than the liquid phase velocity in the surrounding electrolyte. In order to investigate the impact of the wake on the mass transfer near the electrode, wall normal velocities and their fluctuations were analyzed over a time period of $1.25 \mathrm{~s}$ (100 images) before and after the bubble appeared (see Fig.6). It can be seen from the left hand side of Fig. 6 that the bubble curtain entrains fluid when no large bubble is present. However, both the mean velocities and their fluctuations are fairly low. Although the total entrainment is almost not affected by the wake of the large bubble (not illustrated), the fluid is now entrained and ejected section-wise as shown on the right hand side of Fig. 6, which could enhance the convective mass exchange

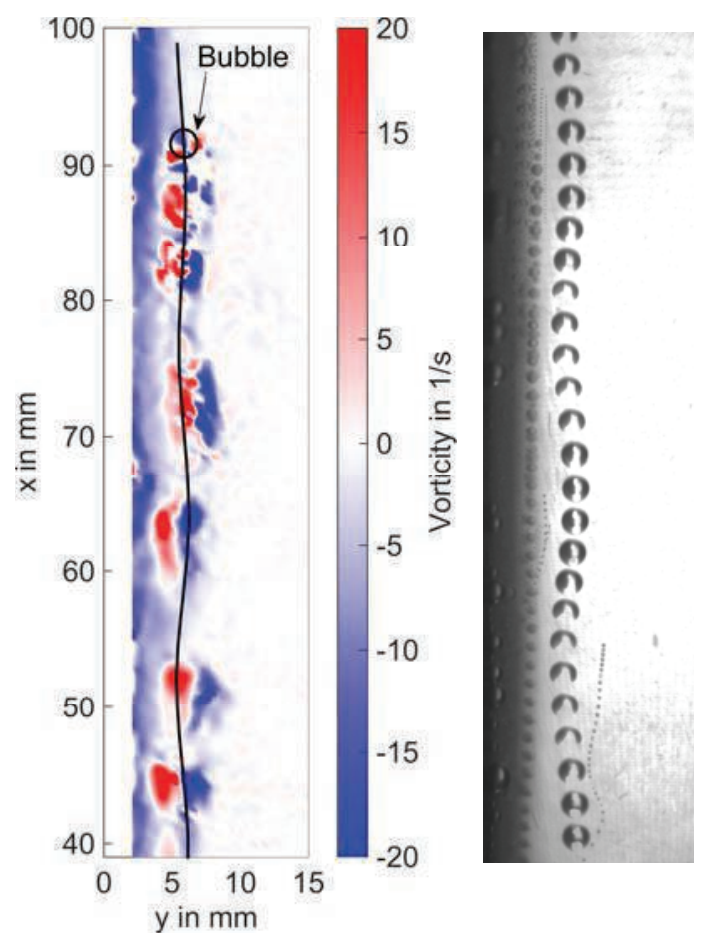

Fig. 5. Instantaneous vorticity field with the position and trajectory of a large oscillating bubble with $d=2$ $\mathrm{mm}$ for a current density of $j=143 \mathrm{Am}^{-2}$ (left) and combined series of corresponding bubble shadow images (right).

of the reaction products in the vicinity of the electrode and the fresh solution in the electrode gap. More importantly, the velocity fluctuations are considerably higher compared to the case without any large bubble. Consequently, the presence of large rising bubbles in the vicinity of the electrode can be beneficial to enhance the mass transfer near the electrodes and thus improve the performance of the hydrogen evolution process.

\section{Conclusions}

The measurement accuracy of PIV and PTV is limited near the gas-evolving electrode as the result of the large light absorption in this region. It was shown that sophisticated PTV techniques can be applied to obtain more precise measurements closer to the electrode. A more powerful laser and a higher optical magnification should be taken into consideration in order to further improve the near-wall measurement accuracy in future experiments. Altogether, the applied PTV approach allowed for a better characterization of the near-wall fluid velocities, which is essential to understand the effect of convection on the detachment of bubbles from the electrode surface. Future work will concentrate on the near-wall effect of Lorentz forces, which can be generated by superimposing magnetic fields. The application of Lorentz forces can lead to an accelerated removal of the hydrogen 

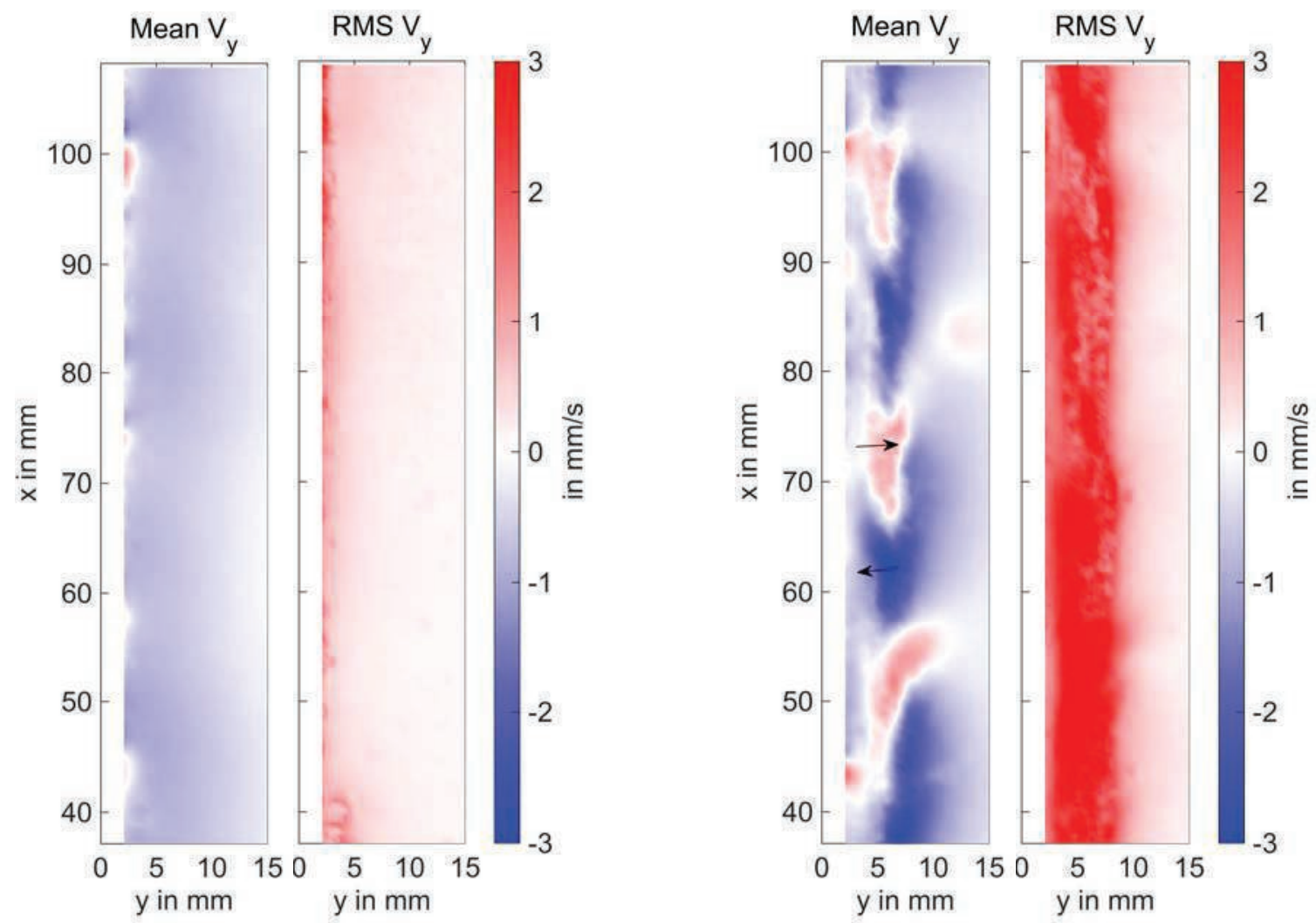

Fig. 6. Mean and RMS values of wall normal velocity component over a period of $1.25 \mathrm{~s}$ (100 images) before (left) and after (right) appearance of a single large bubble with $d=2 \mathrm{~mm}$ (current density $j=143 \mathrm{Am}^{-2}$ ). The bubble needed approximately $0.3 \mathrm{~s}$ to move through the measurement window. The biased PIV area close to the electrode was removed.

bubbles from the electrode surface and, consequently, improve the efficiency of the process.

In addition, the effect of large rising bubbles on the near-wall flow was investigated. It was shown that the presence of a single bubble can strongly affect the near-wall velocities and their fluctuations and thus enhance the mass transfer near the electrode and presumably improve the efficiency of the electrolyzer.

\section{Acknowledgements}

Financial support from the DFG under the grant number Cl 185/3 "Kontrollierte elektrochemische Energieumwandlung durch oberflächennahe Strömungsbeeinflussung" is gratefully acknowledged.

\section{References}

[1] P. Boissonneau, P. Byrne, An experimental investigation of bubble-induced free convection in a small electrochemical cell, Journal of Applied Electrochemistry 30, 767-775 (2000), doi: 10.1023/A:1004034807331

[2] C. Cierpka, B Lütke, C.J. Kähler, Higher order multiframe Particle Tracking Velocimetry. Experiment in Fluids 54, 1533 (2013), doi: $10.1007 / \mathrm{s} 00348-013-1533-3$
[3] J. Duncan, D. Dabiri, J. Hove, M. Gharib, Universal outlier detection for particle image velocimetry (PIV) and particle tracking velocimetry (PTV) data, Measurement Science and Technology 21, 057002, (2010), doi: 10.1088/0957-0233/21/5/057002

[4] D. Fernández, P. Maurer, M. Martine, J.M.D. Coey, M. Möbius, Bubble Formation at a GasEvolving Microelectrode, Langmuir 30, 1306513074 (2014), doi: 10.1021/la500234r

[5] J.A. Koza. S. Mühlenhoff, P. Żabiński, P.A. Nikrityuk, K. Eckert, M. Uhlemann, A. Gebert, T. Weier, L. Schultz, S. Odenbach, Hydrogen evolution under the influence of a magnetic field, Electrochimica Acta 56, 2665-2675 (2011), doi: 10.1016/j.electacta.2010.12.031

[6] K. Ohmi, H.Y. Li, Particle-tracking velocimetry with new algorithms, Measurement Science and Technology 11, 603-616 (2000), doi: $10.1088 / 0957-0233 / 11 / 6 / 303$

[7] T. Weier, S. Landgraf, The two-phase flow at gas-evolving electrodes: Bubble-driven and Lorentz-force-driven convection, The European Physical Journal Special Topics 220, 313-322 (2013), doi: 10.1140/epjst/e2013-01816-1 